\title{
A Validation Metrics Based Model \\ Calibration Applied on Stranded Cables
}

Daniel Alves Castello

castello@mecanica.coppe.ufrj.br Universidade Federal do Rio de Janeiro - UFRJ Dept. of Mechanical Engineering, Poli/COPPE 21945-970 Rio de Janeiro, RJ, Brazil

\section{Carlos Frederico Trotta Matt}

cfmatt@cepel.br

Centro de Pesquisas de Energia Elétrica - CEPEL

Dept. of Lines and Substations

21941-590 Rio de Janeiro, RJ, Brazil

The present work is aimed at building a computational model for a typical stranded cable based on the basic principles of Verification and Validation. The model calibration and model tracking are guided based on a pool of validation metrics suitable for data which are commonly used in structural dynamics. The estimator used for the associated inverse problem is the Maximum a Posteriori estimator and the parameter estimation process is performed sequentially over experiments. Experimental tests have been performed at CEPEL's (Electric Power Research Center) laboratory span with the overhead conductor Grosbeak in order to provide the measured data. The predictive capacity of the computational model is assessed by means of frequency-and time-domain validations through FRFs, band limited white-noise and sine sweep excitations. We also present novel and reliable estimates for the bending stiffness and damping parameters of a widely used transmission line conductor.

Keywords: model calibration, validation metrics, maximum a posteriori, stranded cables, bending stiffness

\section{Introduction}

The use of computational models (CM) in different areas of engineering and applied sciences has become normal in industry and academia. CM have broadly been used for preliminary design, optimization, decision-making, predictions and so on. Such increasing reliance on computer model predictions has naturally led to the nucleation and development of the Verification and Validation (V\&V) field (AIAA, 1998; ASME, 2006).

The AIAA (2008) defines validation as the process of determining the degree to which a model is an accurate representation of the real world from the perspectives of the intended uses of the model. In other words, validation can be defined as the act of quantifying the credibility of a model to represent some phenomena of interest (Sornette et al., 2008). Based on this philosophy some strategies have been proposed in order to determine a credibility level for a computational model (ASME, 2006). A literature review presents some recent articles based on the basic principles of $\mathrm{V} \& \mathrm{~V}$ applied in different areas. Liang et al. (2010) present some methodologies to calibrate power loads and validate power distribution system models. The authors use information of historical loads and measurement data and they also use the calibrated models to generate scenarios for predictions. Greenwald (2010) presents a comprehensive text containing several information about V\&V. Greenwald (2010) emphasizes the usefulness of $\mathrm{V} \& \mathrm{~V}$ for physics of plasmas inasmuch as such field encompasses different ranges of temporal and spatial scales, nonlinearities and extreme anisotropy. Hemez et al. (2010) propose and define some desired characteristics for what is called predictive maturity. According to those authors, predictive maturity is a powerful quantitative tool that could guide decision makers to allocate resources for experimental tests and code development. The quantitative metrics presented by those authors can be used to track model progress as additional information becomes available. Those authors presented detailed analysis for a computational model based on the non-linear Preston-Tonks-Wallace model for plasticity.

Roughly speaking V\&V encompasses several steps such as: (i) proper definition of the intended use of the model, (ii) optimum experiment design and data collection, (iii) model calibration based on parameter estimation, (iv) evaluation of the predictive capacity of the model based on new experimental data, (v) evaluation of the predictive capacity of the model in an environment which provides

Paper received 22 February 2011. Paper accepted 4 July 2011. Technical Editor: Paulo Varoto data more complex than the one used in (iii), (vi) make model revisions based on the result of (v), and (vii) going back to (iii) depending on the result obtained in (v) and (vi). The sequence of the seven steps just presented clearly states that the procedure of calibrating a set of parameters of a chosen model based on experimental data does not assure its predictive capability. Within a $\mathrm{V} \& \mathrm{~V}$ program, calibration can be considered as one of its essential steps.

Model calibration is accomplished based on an inverse problem formulation associated to a system under study and it is based on information both from model predictions and measured data. A keypoint to be considered is that despite the fact that both validation and calibration are processes built on experimental data, calibration is not validation. Although calibration of a model may indicate the model's data fitting ability it does not assure its predictive capabilities (ASME, 2006). Its predictive capabilities should be assessed based on quantitative comparisons between model predictions and measured quantities. The level of complexity associated to these quantitative comparisons can be increased according to a range of operational parameters and different environmental conditions (Hemez et al., 2010). Within a V\&V program, the use of validation metrics is a key-tool to determine these predictive capabilities.

Oberkampf and Barone (2006) recommend some features to define metrics to quantify the agreement between computational and experimental responses. Those authors state that the functional form of the metric is not absolute or unique; it should only measure the agreement between computational results and experimental data in such a way that positive and negative errors cannot cancel. Those authors also emphasize that such metrics should take uncertainties in experimental data and in model predictions whenever it is possible. The metric proposed in Oberkampf and Barone (2006) is based on the statistical concept of confidence intervals. In Oberkampf and Barone (2006) it is also presented three applications where the uncertainties in the measurement are taken into account for the computation of the proposed validation metric. In Liang et al. (2010) it is presented an accuracy index denoted as overall accuracy index which takes into account the ratio between predictions and measurements in a global fashion. Those authors also take into account the stochastic behavior of measured data and also propose an analysis of such index based on the concept of confidence interval. Schwer (2007) proposes a metric suitable to be used with time domain data containing characteristics commonly encountered in structural dynamics signals. 
The general purpose of the present work is to build a simple computational model for a typical stranded cable commonly used in the design of energy transmission line systems. The model building process is guided by the $\mathrm{V} \& \mathrm{~V}$ basic principles. More specifically, the usefulness of the model and the tracking progress of its reliability level are assessed by means of a pool of validation metrics. For this analysis it is considered experimental data both in frequency and time domains collected at CEPEL's (Brazilian Electric Power Research Center) laboratory span for the ACSR cable Grosbeak. For the parameter estimation process it is used a sequential estimation process based on the Maximum a Posteriori estimator. At least in principle, the analysis presented by the authors can be extended to different types of models for vibrating systems. Moreover, we expect that the parameters estimated for the specific stranded cable used in the work can be used for computational prediction-based decisions of the specific cable that was analyzed.

The article presents the following sections: Introduction, Mechanical Vibrations of Stranded Cables: Mathematical Modeling, Inverse Problem, Model Validation, Experimental Set-Up, Results and Final Remarks.

\section{Nomenclature}

APCC = amplitude-phase correlation coefficient

$C_{S G}=$ Sprague and Geers' comprehensive error factor

$D=$ conductor nominal diameter, $m$

$E \quad=$ Young modulus, $P a$

EI = conductor bending stiffness, $\mathrm{Nm}^{2}$

$f \quad=$ probability density function

$F(x, t)=$ external excitation, $\mathrm{Nm}^{-1}$

$\mathbf{H}=$ vector containing frequency response functions

$I=$ cross-section area moment of inertia, $\mathrm{m}^{4}$

$\mathbf{J} \quad=$ sensitivity matrix

$L \quad=$ span length, $m$

$M_{S G}=$ Sprague and Geers' magnitude error metric

$N_{f} \quad=$ number of frequency data points

$N_{s} \quad=$ number of sensors

p = vector of unknown parameters

$P_{S G}=$ Sprague and Geers' phase difference metric

$S_{M A P}=$ maximum a posteriori objective function

$t \quad=$ time, $s$

$T=$ tensile load, $N$

$\mathbf{V}=$ parameter covariance matrix

$\mathbf{W}=$ error covariance matrix

$x \quad=$ axial coordinate, $m$

$y(x, t)=$ conductor transverse displacement, $m$

\section{Greek Symbols}

$\alpha \quad=$ aerodynamic damping coefficient, $\mathrm{Nsm}^{-2}$

$\xi \quad=$ material damping factor, $\mathrm{Pa} \cdot \mathrm{s}$

$\mu=$ conductor mass per unit length, $\mathrm{kg} / \mathrm{m}$

$\sigma_{n} \quad=$ standard-deviation of the measured data

$\sigma_{p 1}=$ standard-deviation of the unknown parameter $p_{1}$

$\sigma_{p 2}=$ standard-deviation of the unknown parameter $p_{2}$

$\sigma_{p 3}=$ standard-deviation of the unknown parameter $p_{3}$

$\omega=$ circular frequency, $\mathrm{rad} / \mathrm{s}$

$\Delta \mathbf{p}^{k}=$ parameter increment at $k^{\text {th }}$ iteration

\section{Subscripts/Superscripts}

$$
\begin{aligned}
& \text { est, } e=\text { estimated } \\
& \text { exp, } x=\text { experimental } \\
& \text { pr } \quad=\text { prior distribution } \\
& H \quad=\text { Hermitian operator } \\
& T \quad=\text { transpose operator }
\end{aligned}
$$

\section{Mechanical Vibrations of Stranded Cables: Mathematical Modeling}

Stranded cables are structural components which possess several applications. This type of component can be used, for example, in cable stayed bridges (Sih et al., 2008) as well as in transmission line systems of electric energy (Hagedorn, 1982; Hagedorn et al., 1987). For the present work we decided to build a model for a typical stranded cable which is commonly used in transmission line systems. This model building is based on the principles of $\mathrm{V} \& \mathrm{~V}$. Hence, some descriptive information concerning physical characteristics of this system, its operational environment and the intended use of its computational model will be provided throughout this section.

The majority of overhead conductors employed in highvoltage transmission lines are composed of steel and aluminum wires helically wrapped around a central core. They are commonly referred to as ACSR or Aluminum Conductor Steel Reinforced. In the field, overhead conductors are strung to high tensile loads and their ends are clamped at the suspension towers. These structures, also referred to as cables or helical strands, find applications in other fields such as civil and ocean engineering (for example, in bridges, towers and masts) due to their high strength. An important feature of such structures is their low bending stiffness (Cardou, 2006). Cardou (2006) provides an excellent literature review of various mechanical models proposed for circular crosssection wire strands aimed at computing their bending stiffness. The first works about this subject rely on a different set of simplifying assumptions, what leads to a more or less accurate evaluation of the bending stiffness. Most of the works cited by Cardou (2006) approaches the problem of estimating some cable parameters by means of static analysis and dynamic analysis based solely on its natural frequencies. The damping parameters of these structures are not discussed by Cardou (2006).

Owing to the complex geometry of a typical overhead conductor under bending, the majority of the theoretical models available in the literature consider such a complex mechanical structure as a continuous homogeneous system (Claren and Diana, 1969; Dhotarad et al., 1978; Hagedorn, 1982; Diana et al., 2000; Barbieri et al., 2004; Matt and Castello, 2007; Castello and Matt, 2007). The simplest models treat the conductors as homogeneous taut strings without bending stiffness, while the more sophisticated ones consider them as homogeneous Euler-Bernoulli beams with constant bending stiffness. Although being a common practice, it does not mean that such equivalent homogeneous models are suitable to describe the dynamic behavior of a transmission line conductor. To the knowledge of the authors, no previous work has attempted to assess the validity of equivalent homogeneous beam models when applied to a transmission line conductor. Hence, a major contribution of the current work is to assess the predictive capabilities of an equivalent homogeneous beam model based on quantitative comparisons between predicted and observed responses of a typical conductor in both time and frequency domains. The beam model seems to be more appropriate to describe the mechanical vibrations of overhead conductors; nevertheless, two problems should be highlighted.

The first problem is that there is a great uncertainty concerning their bending stiffness. The common engineering practice is to choose a constant value for the bending stiffness within the range defined by the minimum and maximum theoretical values. The minimum theoretical value is obtained by considering the conductor as a bundle of individual wires and by assuming that the wires are free to move relative to each other (full slip behavior) (Cardou, 2006). The maximum theoretical value is obtained by considering the conductor as a bundle of individual wires and by assuming that 
the contact pressure among the wires is high enough to prevent their relative motions (full stick behavior) (Cardou, 2006). For typical overhead conductors, the maximum and minimum values may differ by several orders of magnitude; for example, for the ACSR conductor Grosbeak investigated in the current work, the minimum and maximum values are, respectively, $28 \mathrm{Nm}^{2}$ and $1027 \mathrm{Nm}^{2}$. The actual bending stiffness of a typical conductor lies within the range defined by the minimum and maximum theoretical values. During its bending vibrations there may be relative movements between its constituent wires, movements which are constrained by friction among them.

The second one is concerned with damping estimation for stranded cables. Barbieri et al. (2004) estimate the modal damping ratios of a transmission line conductor. In their work they assume the conductor as a homogeneous Euler-Bernoulli beam and estimate the modal damping ratios associated with the first five modes of the Ibis conductor. They also estimate the parameters of a proportional damping matrix adopted for a reduced model (Friswell et al., 1995) of the cable. Their results encompass two different tensile loads and three different lengths of the analyzed cable. An important conclusion of their work is that the estimated modal damping ratios are inversely proportional to the cable tensile load. However, they did not estimate the bending stiffness of the analyzed cable. Kim and Park (2007) approached the problem of estimating cable tension forces based on the measured natural frequencies. Kim and Park (2007) consider the effect of the bending stiffness of the cables and also the sag-extensibility effect. They formulate the inverse problem in a way that it is possible to estimate simultaneously the bending stiffness, the axial rigidity and the cable tensile load. They present results based on numerical simulations and also based on experimental data for cables used in bridges. An important conclusion presented by Kim and Park (2007) is that the estimated bending stiffness was nearly proportional to the cable tensile load. However, they did not take damping into account in their analysis.

Based on the previous information, the authors highlight the interest in obtaining a suitable simple mathematical model for a typical transmission line cable based on the basic principles of $\mathrm{V} \& \mathrm{~V}$ such that: (i) experimental data commonly obtained by dynamic testing can be used for the model building process; (ii) the model calibration considers the estimation of stiffness and damping parameters at the same time; (iii) the model could be used for transient analysis and (iv) the model is suitable for model based optimization strategies.

In the present work a typical conductor is modeled as an equivalent homogeneous Euler-Bernoulli beam with constant bending stiffness and subjected to a constant tensile load. Disregarding both the rotary inertia and sag extensibility, a simple model for the system may be described by the following governing equation (Matt and Castello, 2007; Castello and Matt, 2007):

$$
E I \frac{\partial^{4} y}{\partial x^{4}}-T \frac{\partial^{2} y}{\partial x^{2}}+\xi I \frac{\partial}{\partial t}\left(\frac{\partial^{4} y}{\partial x^{4}}\right)+\alpha \frac{\partial y}{\partial t}+\mu \frac{\partial^{2} y}{\partial t^{2}}=F(x, t) .
$$

where $F(x, t)$ stands for the external excitation; $\mu$ denotes the mass per unit length of the conductor; the fourth term on the left-hand side of Eq. (1) stands for a viscous-like aerodynamic damping; the parameter $\alpha$ is the equivalent aerodynamic damping coefficient; $E$ is the Young modulus and the third term on the left-hand side of Eq. (1) represents, in the equivalent homogeneous beam model, the energy dissipation mechanism associated with the inter-strand friction among the wires of a typical conductor, i.e., $\xi$ is a material damping factor.

Concerning the simple damping models chosen to describe energy dissipation mechanisms, in Eq. (1), the following remarks should be highlighted. The first one is that the aerodynamic damping (i.e., the energy dissipation due to friction between the vibrating conductor and surrounding air) is represented by a linear viscous damping model (the fourth term on the left-hand side of Eq. (1)). The majority of previous works neglects aerodynamic damping, although researchers strongly recommend corrections on the measured energy dissipated by a transmission line conductor in order to account for it (Rawlins, 1983). The second one is that the conductor self-damping is represented by a linear damping model derived from the Kelvin-Voigt constitutive relationship and for which the damping force becomes directly proportional to the time rate of change of conductor curvature (the third term on the lefthand side of Eq. (1)); one should note that the friction among the conductor wires depends somehow on the time rate of change of its curvature during bending vibrations. Although we have not considered hysteretic damping model in Eq. (1), it is well-known that the majority of works that takes into account the conductor selfdamping represent it by linear hysteretic damping models, in which the damping force is directly proportional to conductor vibrating velocity and inversely proportional to the excitation frequency. However, it is well-known that such linear hysteretic damping model has two flaws. Firstly, it violates the causality principle (Crandall, 1970; Adhikari, 2000) and, secondly, it can be used only for single-frequency harmonic excitations. Finally, the equivalent homogeneous beam model mathematically described by Eq. (1) may naturally be coupled with the fluid dynamics equations in order to simulate the fluid-structure interaction problem governing the Aeolian vibrations (Rawlins, 1979; Cigré, 1989; Meynen et al., 2005) on a transmission line conductor in a way similar to the one presented by Wang et al. (2001).

\section{Direct problem}

The direct problem consists in finding the solution of Eq. (1), satisfying the appropriate boundary and initial conditions, with the conductor parameters $E I, \alpha$ and $\xi I$, and the excitation $F(x, t)$ being known. All the remaining parameters appearing in Eq. (1) are assumed to be known. Several analytical techniques and numerical methods may be used to solve the direct problem. Here, the direct problem is solved through the finite-element method (Hughes, 2000). The details of the finite-element solution of the aforementioned direct problem may be encountered elsewhere (Matt and Castello, 2007; Castello and Matt, 2007).

\section{Inverse Problem: Parameter Estimation}

For the inverse problem of parameter estimation considered here, the conductor bending stiffness $E I$, the aerodynamic damping coefficient $\alpha$ and the internal dissipation factor $\xi I$ are regarded as being unknown. The additional information used to estimate these parameters are the complex frequency response functions measured at prescribed locations $x=x_{a}, a=1,2, \ldots, N_{s}$, along the conductor and at circular frequencies $\omega_{b}, b=1,2, \ldots, N_{f}$, where $N_{s}$ is the number of sensors and $N_{f}$ is the number of frequency data.

For the parameter estimation process we consider that the unknown vector $\mathbf{p}$ is a random vector. Therefore, based on the Bayes' rule for conditional probabilities, we can write

$$
f\left(\mathbf{p} \mid \mathbf{H}^{\exp }\right)=\frac{f\left(\mathbf{H}^{\exp } \mid \mathbf{p}\right) f_{p r}(\mathbf{p})}{f\left(\mathbf{H}^{\exp }\right)}
$$

where $f\left(\mathbf{p} \mid \mathbf{H}^{\text {exp }}\right)$ corresponds to the posterior probability density function of $\mathbf{p}$ given the measured FRF $\mathbf{H}^{\exp } ; f\left(\mathbf{H}^{\exp } \mid \mathbf{p}\right)$ corresponds to the likelihood function; $f_{p r}(\mathbf{p})$ corresponds to the a priori probability 
density function of $\mathbf{p}$ and $f\left(\mathbf{H}^{\text {exp }}\right)$ corresponds to a normalizing factor. Adopting the hypothesis that the measurement errors are Gaussian distributed and that our current state of knowledge about $\mathbf{p}$, which is represented by the prior $f_{p r}(\mathbf{p})$, can also be represented by a multivariate Gaussian distribution, we can formulate our inverse problem based on the search for the point $\hat{p}$ which maximizes $f\left(\mathbf{p} \mid \mathbf{H}^{\text {exp }}\right)$. Owing to the hypotheses previously mentioned, maximization of $f\left(\mathbf{p} \mid \mathbf{H}^{\exp }\right)$ is equivalent to the minimization of the Maximum a Posteriori estimator $S_{M A P}$, given as

$$
\begin{aligned}
S_{M A P}(\mathbf{p}) & =\left[\mathbf{H}^{\exp }-\mathbf{H}^{e s t}(\mathbf{p})\right]^{T} \mathbf{W}\left[\mathbf{H}^{\exp }-\mathbf{H}^{e s t}(\mathbf{p})\right] \\
& +\left[\mathbf{p}_{\mu}-\mathbf{p}\right]^{T} \mathbf{V}^{-1}\left[\mathbf{p}_{\mu}-\mathbf{p}\right]
\end{aligned}
$$

where $\mathbf{H}^{\text {est }}(\mathbf{p})$ and $\mathbf{W}$ denote, respectively, the model FRF and the inverse of error covariance matrix; $\mathbf{p}_{\mu}$ and $\mathbf{V}$ denote, respectively, the mean value and the covariance matrix of the unknown parameters based on our prior state of knowledge about p. By assuming that the measurement errors are additive, uncorrelated and normally distributed, the weighting matrix $\mathbf{W}$ is a diagonal matrix with the reciprocal of the covariance of the measurements, $1 / \sigma_{n}^{2}, n=1,2, \ldots, N_{f} \times N_{s} \times 2$, on its diagonal (Orlande, 2002). The factor 2 appears because both real and imaginary parts of the measured FRFs are taken into account, as explained later on this section. The iterative procedure for the minimization of $S_{M A P}(\mathbf{p})$, given by Eq. (3), may be written in the form (Orlande, 2002)

$$
\Delta \mathbf{p}^{k}=\left[\mathbf{J}^{k^{T}} \mathbf{W} \mathbf{J}^{k}+\mathbf{V}^{-1}\right]^{-1}\left\{\mathbf{J}^{k} \mathbf{W}\left[\mathbf{H}^{\text {exp }}-\mathbf{H}^{e s t}\left(\mathbf{p}^{k}\right)\right]+\mathbf{V}^{-1}\left(\mathbf{p}_{\mu}-\mathbf{p}^{k}\right)\right\}
$$

The estimation process will be considered sequentially over experiments. We take the experimental data in two disjoint frequency bands. Initially we start the estimation process based on the experimental information within the lower frequency band and taking into account the fact that our a priori information about $\mathbf{p}$ is not reliable. This is accomplished by considering the covariance matrix $\mathbf{V}$ composed of large components. Once we have obtained an estimate for $\mathbf{p}$ based on the experimental data of the first frequency band, we take this information into account as the a priori information for the second set of experimental data. Hence, we build up $S_{M A P}(\mathbf{p})$ and perform once again the estimation process based on the experimental data associated with the second frequency band. We decided to approach the inverse problem based on a sequentially staggered way due to the fact that transmission line cables possess high modal density spectra and that the frequency range associated to aeolian vibrations, in general, encompasses a great number of modes for this type of structures. Therefore, we consider the experimental data in two disjoint frequency ranges instead of taking into account a great amount of experimental data at the same time for the inverse problem.

It should be remarked that, as the parameter estimation techniques have to deal with experimental data which possess real and imaginary parts, we decided to arrange them in the following fashion:

$$
\mathbf{H}^{q}=\left(\begin{array}{c}
\mathbf{L}^{q}\left(\omega_{1}\right) \\
\vdots \\
\mathbf{L}^{q}\left(\omega_{N_{f}}\right)
\end{array}\right)
$$

where
$\mathbf{L}^{q}\left(\omega_{r}\right)=\left\{\operatorname{Re}\left[H_{1}^{q}\left(\omega_{r}\right)\right], \operatorname{Im}\left[H_{1}^{q}\left(\omega_{r}\right)\right], \ldots, \operatorname{Re}\left[H_{N_{s}}^{q}\left(\omega_{r}\right)\right] \operatorname{Im}\left[H_{N_{s}}^{q}\left(\omega_{r}\right)\right]\right\}^{T}$

where $r=1,2, \ldots, N_{f}$, the superscript $q$ may be equal to $\exp$ or $\operatorname{est}$ and the subscript under the symbol $H$ corresponds to the number of the sensor.

\section{Model Validation}

Once we have performed the parameter estimation process aiming at calibrating the computational model to a set of experimental data a natural question that one may raise is: how accurate are the predictions provided by a computational model? Such question is suitable here, inasmuch as once we have chosen a specific model such as Eq. (1) and estimated its parameters, what level of confidence can we assign to this computational model? This answer can be partially fulfilled by validation processes.

As it has been previously mentioned, the AIAA report (AIAA, 1998) defines validation as the process of determining the degree to which a model is an accurate representation of the real world from the perspectives of the intended uses of the model. In the present work we will track the progress of our model calibration based on basic principles of $\mathrm{V} \& \mathrm{~V}$. More specifically, the calibration process will be guided by some validation metrics. In order to assure that the validation process remains independent of calibration processes, it is imperative to keep their associated experimental data disjoint.

The validation metrics used here should be suitable to quantitatively compare data associated to structural dynamic analysis. Among some possible metrics we decided to use the following validation metrics: (i) the amplitude-phase correlation coefficient (henceforth abbreviated as APCC); (ii) the Sprague and Geers metrics (Schwer, 2007; Sprague and Geers, 2003) and (iii) a point-to-point error norm. The APCC is defined as

$$
\operatorname{APCC}(\omega)=\frac{2 \mathbf{a}^{H}(\omega) \mathbf{b}(\omega)}{\mathbf{a}^{H}(\omega) \mathbf{a}(\omega)+\mathbf{b}^{H}(\omega) \mathbf{b}(\omega)}
$$

where $(\bullet)^{H}$ denotes the Hermitian operator and $\mathbf{a}$ and $\mathbf{b}$ correspond to vectors containing all FRFs measured at the frequency $\omega$. The absolute value of APCC is equal to one if and only if $\mathbf{a}$ and $\mathbf{b}$ possess the same magnitude; otherwise, its absolute value is always less than one. This metric seems appropriate inasmuch as it provides a quantitative comparison at a frequency $\omega$ taking into account a group of FRFs to compose vectors $\mathbf{a}$ and $\mathbf{b}$. For comparisons of time domain signals composed of several frequency components, Oberkampft and Barone (2006) suggest the Sprague and Geers metrics. Sprague and Geers (2003) proposed metrics to quantify magnitude errors, $M_{S G}$, and phase differences, $P_{S G}$; the former is insensitive to phase differences whereas the latter is insensitive to magnitude differences. Sprague and Geers also retained Geers original idea of one number representing the combined magnitude and phase differences, named Comprehensive Error Factor, $C_{S G}$. These metrics are defined in Eqs. (8), (9) and (10):

$$
\begin{aligned}
& M_{S G}=\sqrt{v_{x e} / v_{x x}}-1 \\
& P_{S G}=\frac{1}{\pi} \cos ^{-1}\left(\frac{v_{x e}}{\sqrt{v_{x x} v_{e e}}}\right) \\
& C_{S G}=\sqrt{M_{S G}^{2}+P_{S G}^{2}}
\end{aligned}
$$


where, for simplicity, the (abbreviated) subscripts $(\bullet)_{x}$ and $(\bullet)_{e}$ refer to experimental and estimated quantities, respectively; and $v_{a b}$ is defined as follows:

$$
v_{a b}=\frac{1}{\left(t_{2}-t_{1}\right)} \int_{t_{1}}^{t_{2}} y^{a}(t) y^{b}(t) d t, a, b \in\{x, e\}
$$

where $t_{1}<t<t_{2}$ is the time span of interest for the response history. For further details about Eqs. (8), (9) and (10), the reader should refer to Schwer (2007) where that author analyzed the Sprague and Geers metrics applied to experimental wave-like signals and also presented comparisons of these metrics with opinions of a group of experts in the field.

The third validation metric is a point-to-point error norm $\left|\mathbf{q}^{e s t}\right|_{N}$ which is defined for a generic estimated quantity $\mathbf{q}^{e s t}$ as follows:

$$
\left|\mathbf{q}^{\text {est }}\right|_{N}=\frac{\left(\mathbf{q}^{\text {exp }}-\mathbf{q}^{\text {est }}\right)^{H}\left(\mathbf{q}^{\text {exp }}-\mathbf{q}^{\text {est }}\right)}{\left(\mathbf{q}^{\text {exp }}\right)^{H}\left(\mathbf{q}^{\text {exp }}\right)} .
$$

A natural question at this point is concerned with which values are considered acceptable for a validation metric. Schwer (2007) states that establishing upper limits on acceptable accuracy remains an open topic in the V\&V community. Geers (1984) 'suggests' the following rule-of-thumb guidance on values for his combined metric: "My personal reaction has been that anything below about $20 \%$ is really good. When you get to around $20-30 \%$, it is getting fair. When you get above the $30-40 \%$ range that is rather poor." In the current work, we adopt the $20 \%$ as the upper limit on the Sprague and Geers metrics for an acceptable accuracy. All in all, these pool of metrics enable one to perform some quantitative comparisons between the predictive capacity of a group of models.

As just mentioned, the pool of metrics presented in this section will be used to assess the predictive capabilities of the computational model described in the Mathematical Modeling section. Therefore, once they provide results not in favor of the model, it clearly means that an action must be taken to initiate improvements in some steps of the modeling and/or experimental design. Hence, instead of judging the suitability of a model based solely on data-fitting graphs we will guide our analysis based on the basic principles of $\mathrm{V} \& \mathrm{~V}$.

\section{Experimental Set-Up}

This section is dedicated to describe the experimental set-up. All the experiments have been performed at the laboratory span of the Electric Power Research Center (CEPEL). The transmission line conductor under analysis is the ACSR Grosbeak, whose weight per unit length and nominal diameter are, respectively, $\mu=1.3027 \mathrm{~kg} / \mathrm{m}$ and $D=25.15 \mathrm{~mm}$. The span length used in the tests is $L=51.950 \mathrm{~m}$. The tests are performed for two different tensile loads, namely $16481 \mathrm{~N}(1680 \mathrm{kgf})$ and $21778 \mathrm{~N}$ (2220 kgf). The tensile loads of $1680 \mathrm{kgf}$ and $2220 \mathrm{kgf}$ correspond to approximately $14 \%$ and $19 \%$ of the Grosbeak rated tensile strength (RTS), percentages commonly employed in the field.

The Grosbeak conductor is instrumented with an electrodynamic shaker and with three piezoelectric accelerometers, namely AC1, AC2 and AC3. The electrodynamic shaker employed for the FRF measurements was manufactured by Data Physics, model S-150 with controller DP-V150 and amplifier A-10C-05. The power of the amplifier is $1250 \mathrm{~W}$; the maximum shaker displacement is $25.4 \mathrm{~mm}$ peak-to-peak; the maximum velocity is $1.5 \mathrm{~m} / \mathrm{s}$; the maximum acceleration is $72 \mathrm{~g}$; the nominal forces are $1000 \mathrm{~N}, 650 \mathrm{~N}$ and $1300 \mathrm{~N}$ respectively for sinusoidal, random and shock; and, finally, the frequency range is $2 \mathrm{~Hz}$ to $5 \mathrm{kHz}$. The force transducer used was manufactured by Bruel \& Kjäer, model 8230-002 with nominal sensitivity $2.41 \mathrm{mV} / \mathrm{N}$. The three accelerometers used were manufactured by Bruel \& Kjäer, model Deltatron 4519-001 with 1 gr mass. The force transducer and the three accelerometers are IEPE; their frequency ranges encompass $5 \mathrm{~Hz}$ to $6 \mathrm{kHz}$, according to the manufacturer's specification.

Figure 1 shows a sketch of the experiment together with the accelerometer positions. The dimensions shown in Fig. 1 are $\ell_{1}=1.4 \mathrm{~m}, \ell_{2}=0.7 \mathrm{~m}$ and $\ell_{3}=1.6 \mathrm{~m}$. It is worthwhile to mention that the electrodynamic shaker is located at the same position as the accelerometer AC3, i.e., the position given by $x=\ell_{3}$ is the driving point of the conductor.

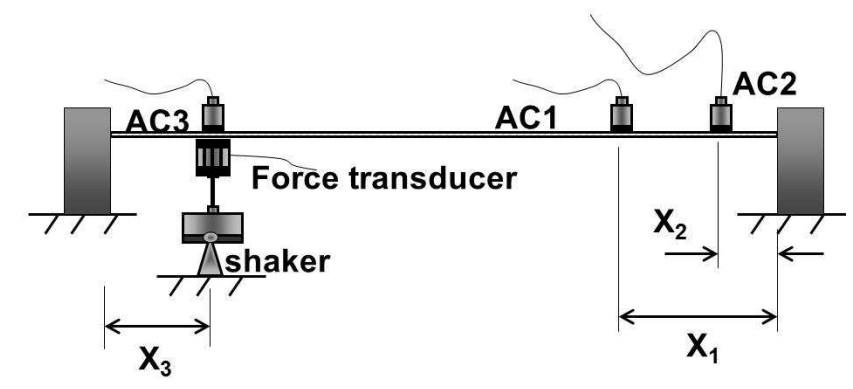

Figure 1. Experimental Set-up.

The signals from the electrodynamic shaker and from the three piezoelectric accelerometers are read and recorded by PULSE acquisition data system from Bruel and Kjäer, which, in turn, computes the desired frequency response functions. For each tensile load, we decided to measure the FRFs for two different frequency bands, as shown in Table 1. The frequency bands shown in Table 1 are within the frequency range expected for aeolian vibrations in the field. All the FRFs are measured with 801 equally spaced frequency points. Such a high frequency resolution becomes necessary in both frequency bands in order to capture the closely-spaced natural frequencies of the conductor and to obtain well-defined peaks in the FRFs. For validation processes, time-domain acceleration data are also measured for band limited white-noise and sine sweep excitations, as indicated in Tables 2 and 3.

Table 1. Characteristics of the measured FRFs during tests performed at CEPEL's laboratory span.

\begin{tabular}{cccc}
\hline $\begin{array}{l}\text { Frequency } \\
\text { range }(\mathrm{Hz})\end{array}$ & $\begin{array}{c}\text { Frequency } \\
\text { resolution } \\
\text { (points) }\end{array}$ & Averages & Accelerometers \\
\hline$[5,17.5]$ & 801 & 30 & $\begin{array}{c}\mathrm{AC} 1, \mathrm{AC} 2 \text { and } \\
\mathrm{AC} 3\end{array}$ \\
{$[17.5,30]$} & 801 & 30 & $\mathrm{AC} 1, \mathrm{AC} 2$ and \\
$\mathrm{AC} 3$
\end{tabular}

Table 2. Time-domain signals recorded for the conductor Grosbeak under the tensile load $T=16481 \mathrm{~N}$.

\begin{tabular}{lllll}
\hline $\begin{array}{l}\text { Excitation } \\
\text { signal }\end{array}$ & $\begin{array}{l}\text { Frequency } \\
\text { band }[\mathrm{Hz}]\end{array}$ & $\begin{array}{l}\text { Time } \\
\text { span } \\
{\left[t_{0}, t_{f}\right] \mathrm{s}}\end{array}$ & $\begin{array}{l}\text { Sampling } \\
\text { frequency } \\
f_{s}[\mathrm{~Hz}]\end{array}$ & Accelerometers \\
\hline $\begin{array}{l}\text { White } \\
\text { noise }\end{array}$ & {$[5,30]$} & {$[0,15]$} & 256 & $\begin{array}{l}\mathrm{AC} 1, \mathrm{AC} 2 \text { and } \\
\mathrm{AC} 3\end{array}$ \\
\hline
\end{tabular}


Table 3. Time-domain signals recorded for the conductor Grosbeak under the tensile load $T=21778 \mathrm{~N}$.

\begin{tabular}{|c|c|c|c|c|}
\hline $\begin{array}{l}\text { Excitation } \\
\text { signal }\end{array}$ & $\begin{array}{l}\text { Frequency } \\
\text { band }[\mathrm{Hz}]\end{array}$ & $\begin{array}{l}\text { Time } \\
\text { span } \\
{\left[t_{0}, t_{f}\right] \mathrm{s}}\end{array}$ & $\begin{array}{l}\text { Sampling } \\
\text { frequency } \\
f_{s}[\mathrm{~Hz}]\end{array}$ & Accelerometers \\
\hline $\begin{array}{l}\text { Sine } \\
\text { sweep }\end{array}$ & {$[5,20]$} & {$[0,15]$} & 256 & $\begin{array}{l}\mathrm{AC} 1, \mathrm{AC} 2 \text { and } \\
\mathrm{AC} 3\end{array}$ \\
\hline $\begin{array}{l}\text { White } \\
\text { noise }\end{array}$ & {$[5,17.5]$} & {$[0,15]$} & 256 & $\begin{array}{l}\mathrm{AC} 1, \mathrm{AC} 2 \text { and } \\
\mathrm{AC} 3\end{array}$ \\
\hline
\end{tabular}
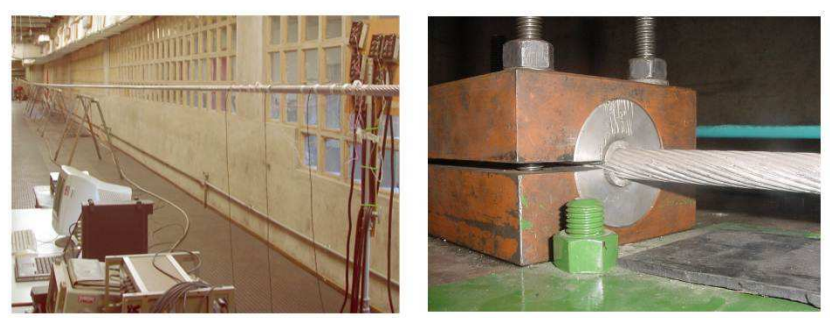

Figure 2. Photographs of the experimental set-up: (left) general overview of the CEPEL's laboratory span and (right) detailed view of conductor's clamp.

Figure 2 shows photographs of the CEPEL's laboratory span. The photograph on the left side of Fig. 2 gives a general overview of the experimental set-up. The photograph on the right side of Fig. 2 gives a detailed view of one conductor end, from which one can see both the conductor clamp and the rigid block fixed on the floor. The other conductor end is identical. From the photograph one may conclude that the clamps restrict both the conductor displacement and rotation; hence, the direct problem previously described is solved for clamped-clamped boundary conditions, i.e.,

$$
y(0, t)=y(L, t)=0
$$

and

$$
\frac{\partial y}{\partial x}(0, t)=\frac{\partial y}{\partial x}(L, t)=0 .
$$

To close up this section, the authors would like to remark that the maximum displacement at the span midpoint during static equilibrium was measured for the two tensile loads tested. The maximum sag-to-span ratio was less than $0.6 \%$. Since the sag-tospan ratios found in the experiments were very lower than $1 / 8$, we decided to neglect its effect on the governing equation of motion, as recommended by Irvine (1981).

\section{Results}

The goals of this section are two-fold. First, we present the estimates obtained for the bending stiffness and the damping parameters of the Grosbeak conductor based on the measured FRFs. Second, we assess the credibility of the model through the basic principles of a validation process. As it has been previously mentioned, the experimental data associated to the estimation process must be different from the one associated to the validation processes. Here, validation is performed by graphically comparing estimated and measured quantities and by computing the validation metrics previously presented.

The estimation process is performed in two stages; the reasons behind this choice are explained later on this section. In the first stage, only the bending stiffness $E I$ and the aerodynamic damping coefficient $\alpha$ are estimated; the material damping factor $\xi I$ is kept constant and equal to a small value. The first stage takes into account only the measured FRFs within the frequency band of [5,17.5] Hz; EI and $\alpha$ are estimated through the LevenbergMarquardt parameter estimation technique (Özişik and Orlande, 2000). In the second stage, we consider two possible models to be calibrated given a new set of measured information: (i) we still estimate the bending stiffness $E I$ and the aerodynamic damping coefficient $\alpha$ while keeping the material damping factor $\xi I$ constant as for the first stage; and (ii) we also estimate the three unknown parameters ( $E I, \alpha$ and $\xi I$ ) simultaneously. During the second stage, the unknown parameters are estimated through the sequential parameter estimation technique. The second stage takes into account information from both (i) the frequency band $[17.5,30] \mathrm{Hz}$ through the associated FRFs (new data) and (ii) the frequency band $[5,17.5] \mathrm{Hz}$ through the prior information. In other words, the estimated parameters and their covariances obtained in the first stage are used as a priori information for the sequential estimation during the second stage. Beck (2003) defines the two-stage parameter estimation strategy proposed here as Sequential Parameter Estimation Over Experiments. We have at our disposal experimental data in both frequency and time domains for the two tensile loads investigated: $16481 \mathrm{~N}$ and $21778 \mathrm{~N}$. The experimental data in the frequency domain are the measured FRFs whereas the experimental data in the time domain are the acceleration and force signals recorded by the accelerometers and the force transducer. The time-domain signals are recorded for white noise and sine sweep excitations.

Concerning the initial guesses for the unknown parameters, it should be noted that we have reference values only for the bending stiffness: the maximum and minimum theoretical values which for the conductor Grosbeak are, respectively, $E I_{\max }=1027 \mathrm{Nm}^{2}$ and $E I_{\min }=28 \mathrm{Nm}^{2}$ (Cigré, 1989). The minimization of the ordinary least-squares norm is performed for three different initial guesses chosen for $E I, E I^{(0)}=\{28,527,1027\} \mathrm{Nm}^{2}$, and for only one initial guess chosen for $\alpha, \alpha^{(0)}=0.1 \mathrm{Nsm}^{-2}$. The three different initial guesses chosen for $E I$ lead to the same final values for the unknown parameters; hence, only the estimates obtained from the initial guess $E I^{(0)}=527 \mathrm{Nm}^{2}$ are reported in the current work. Preliminary numerical tests were performed in order to check the convergence and accuracy of the finite-element solution of the associated direct problem. Based on these numerical tests, we verify that a finite element mesh with one-hundred elements provides acceptable results for the desired degree of accuracy.

\section{First stage}

The experimental data used in the first stage of the estimation process are the measured FRFs of the accelerometers AC2 and AC3 within the frequency band of $[5,17.5] \mathrm{Hz}$. The material (internal) damping $\xi I$ is kept constant and equal to $10^{-4} \mathrm{Nsm}^{2}$. In order to avoid a parameter vector containing components with very different orders of magnitude, the following parameterization is adopted: $E I=p_{1} \times 10^{3}$ and $\alpha=p_{2}$. The final values for the parameters and their standard deviations are shown in Table 4. As far as we know, the estimates obtained for the bending stiffness and aerodynamic damping coefficient of the conductor Grosbeak are new in the literature.

We start the validation process by comparing the estimated and the measured FRFs for the accelerometer AC1, which was not used in the estimation process. Figure 3 shows the measured (circles) and estimated (continuous line) FRFs (magnitude) for the accelerometer $\mathrm{AC} 1$ in the frequency band $[5,17.5] \mathrm{Hz}$. 

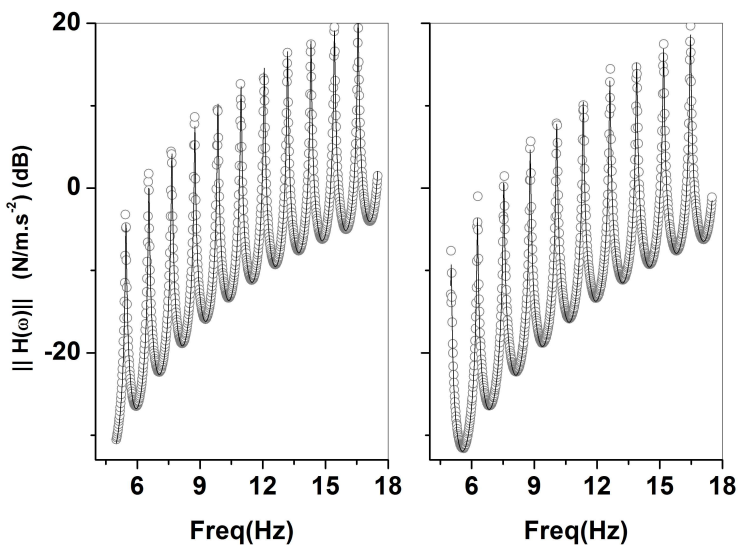

Figure 3. Measured (circles) and estimated (continuous line) FRFs for the accelerometer AC1. On the left $T=16481 \mathrm{~N}$ and on the right $T=21778 \mathrm{~N}$.

Based on the curves indicated on Fig. 3, one may conclude that the equivalent homogeneous beam model with $E I$ and $\alpha$ given in Table 4 and $\xi I=10^{-4} \mathrm{Nsm}^{2}$ reproduces quite well the dynamic behavior of the conductor Grosbeak in the frequency band of [5,17.5] Hz, for both tensile loads. The excellent agreement verified in Fig. 3 may also be viewed from the plot of the APCC validation metric, shown in Fig. 4. From Fig. 4 one may verify that, for the majority of the frequency data points, the corresponding APCC magnitudes are closer to unity. For $T=21778 \mathrm{~N}$, the APCC magnitude lies below 0.80 only for the data point corresponding to the frequency $12 \mathrm{~Hz}$.
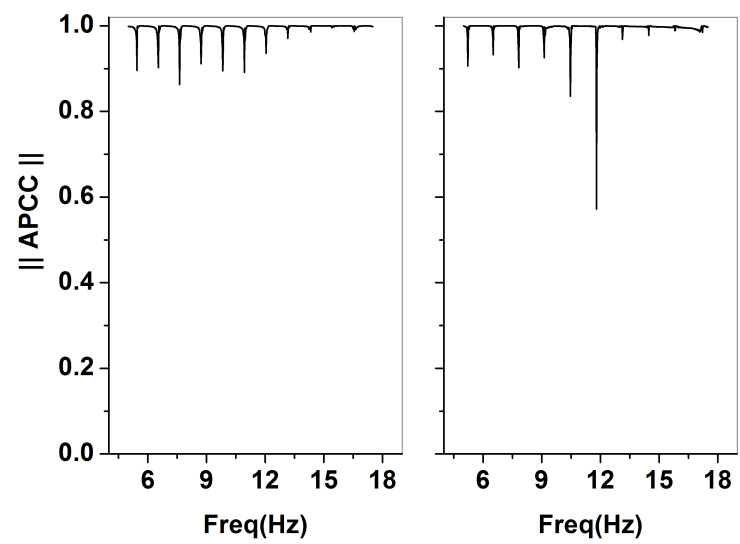

Figure 4. Magnitude of the APCC validation metric for the accelerometer AC1 in the frequency band of $[5,17.5] \mathrm{Hz}$. On the left $T=16481 \mathrm{~N}$ and on the right $T=21778 \mathrm{~N}$.

Table 4. Estimates for the bending stiffness $E I=p_{1} \times 10^{3}$, the aerodynamic damping coefficient $\alpha=p_{2}$ and their corresponding standard deviations for the first stage $\left(\xi I=10^{-4} \mathrm{Nsm}^{2}\right)$.

\begin{tabular}{ccccc}
\hline$T[\mathrm{kgf}]$ & $p_{1}\left[\mathrm{Nm}^{2}\right]$ & $p_{2}\left[\mathrm{Nsm}^{-2}\right]$ & $\sigma_{p_{1}} / \sigma_{n}$ & $\sigma_{p_{2}} / \sigma_{n}$ \\
\hline 1680 & 0.5140 & 0.3189 & 0.002 & 0.010 \\
2220 & 0.7415 & 0.3445 & 0.003 & 0.011 \\
\hline
\end{tabular}

We continue the validation process by comparing the measured and estimated time history accelerations for a white-noise excitation. For $T=21778 \mathrm{~N}$ the excitation encompasses the frequency range [5, 17.5] $\mathrm{Hz}$ (see Table 3) and the measured and estimated time history accelerations of the conductor Grosbeak at the three accelerometer positions are shown in Figs. 5, 6 and 7. The time domain Sprague and Geers metrics were computed for $t \in[8,11] \mathrm{s}$. Nevertheless, for clarity of the figures we decided to plot time domain histories only for $t \in[9,10] \mathrm{s}$.

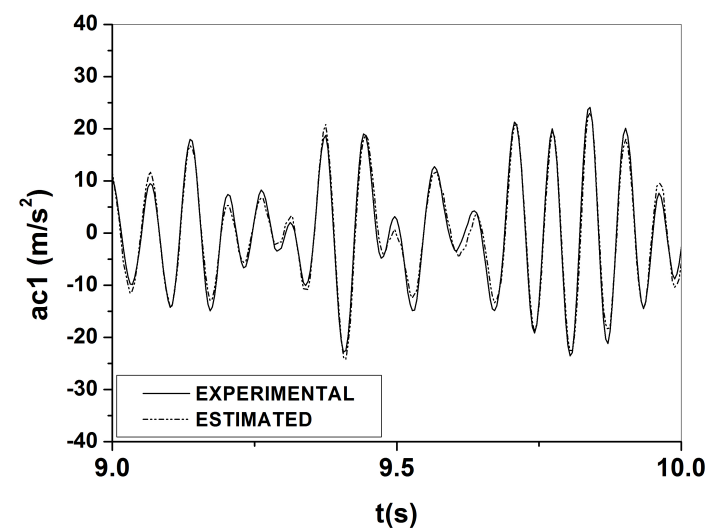

Figure 5. Measured and estimated time history accelerations at the first accelerometer for a white-noise excitation encompassing the frequency band of $[5,17.5] \mathrm{Hz}(T=21778 \mathrm{~N})$.

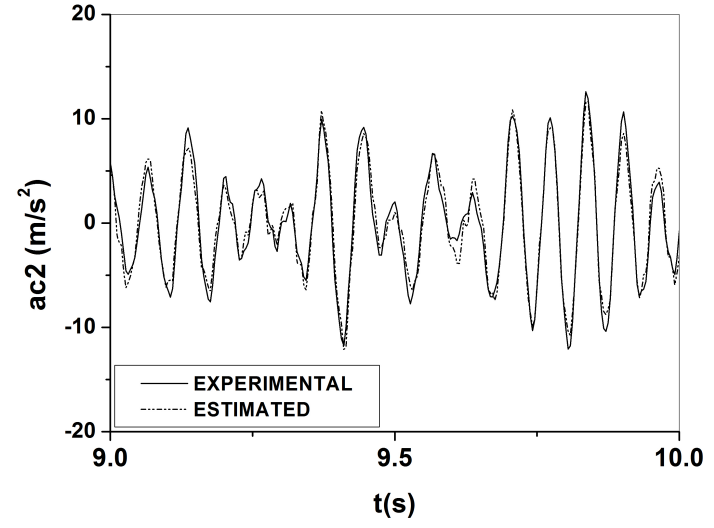

Figure 6. Measured and estimated time history accelerations at the second accelerometer for a white-noise excitation encompassing the frequency band of $[5,17.5] \mathrm{Hz}(T=21778 \mathrm{~N})$.

The estimated time-domain responses shown in Figs. 5, 6 and 7 are obtained from the equivalent homogeneous beam model with $T=21778 \mathrm{~N}, E I=741.5 \mathrm{Nm}^{2}, \alpha=0.3445 \mathrm{Nsm}^{-2}$ and $\xi I=10^{-4}$ $\mathrm{Nsm}^{2}$. The discrete evolution equations of the system are integrated with the Newmark method (Hughes, 2000). The excitation force applied to the beam model is the force signal recorded by the force transducer. From the analysis of Figs. 5, 6 and 7 we conclude that the estimated time-domain accelerations are in excellent agreement with the experimental ones for the three accelerometers. For 
quantitative comparison of these time-domain responses, we also compute in Table 5 the Sprague and Geers metrics.

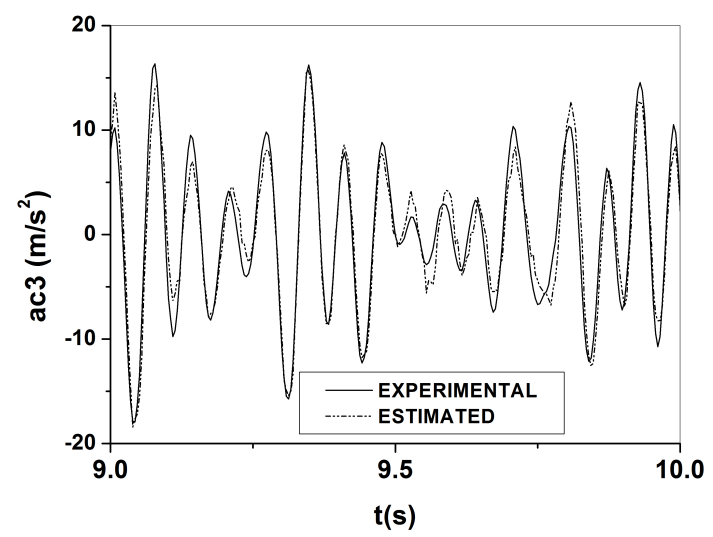

Figure 7. Measured and estimated time history accelerations at the third accelerometer for a white-noise excitation encompassing the frequency band of $[5,17.5] \mathrm{Hz}(T=21778 \mathrm{~N})$.

Table 5. Sprague and Geers validation metrics for comparison between measured and estimated time-domain responses under a white noise excitation encompassing the frequency band of $[5,17.5] \mathrm{Hz}(T=21778 \mathrm{~N})$.

\begin{tabular}{ccccc}
\hline Accelerometer & $M_{S G}$ & $P_{S G}$ & $C_{S G}$ & $\left|\mathbf{q}^{\text {est }}\right|_{N}$ \\
\hline AC1 & 0.0466 & 0.0656 & 0.0805 & 0.2059 \\
AC2 & 0.0618 & 0.0997 & 0.1173 & 0.3082 \\
AC3 & 0.0558 & 0.1358 & 0.1469 & 0.4155 \\
\hline
\end{tabular}

From the analysis of Table 5 we verify that the largest Comprehensive Error Factor, $C_{S G}$, is $14.7 \%$, for the accelerometer AC3. Assuming $20 \%$ as the upper limit on acceptable accuracy for model validation (Schwer, 2007; Geers, 1984), we may thus state that the agreement between prediction and experiment is indeed quite high. Therefore, the set of validation metrics previously presented gives a position in favor of the equivalent homogeneous beam model for $T=21778 \mathrm{~N}$. In principle, one could state that, based on these validation metrics, this model is able to reproduce the dynamic behavior of the system within the frequency range $[5,17.5] \mathrm{Hz}$.

We also compare the measured and estimated time history accelerations under a white-noise excitation for $T=16481 \mathrm{~N}$; in this case, the excitation encompasses the frequency range $[5,30] \mathrm{Hz}$ (see Table 2). Quantitative comparisons among these time-domain responses are presented in Table 6 through the Sprague and Geers' validation metrics.

Table 6. Sprague and Geers validation metrics for comparison between measured and estimated time-domain responses under a white noise excitation encompassing the frequency band of $[5,30] \mathrm{Hz}(T=16481 \mathrm{~N})$.

\begin{tabular}{ccccc}
\hline Accelerometer & $M_{S G}$ & $P_{S G}$ & $C_{S G}$ & $\left.\mathbf{q}^{e s t}\right|_{N}$ \\
\hline AC1 & -0.4580 & 0.2508 & 0.5222 & 1.3422 \\
AC2 & -0.4902 & 0.2570 & 0.5534 & 1.4611 \\
AC3 & -0.5765 & 0.3679 & 0.6839 & 2.1615 \\
\hline
\end{tabular}

Now, the lowest Comprehensive Error Factor $C_{S G}$ is $52.2 \%$, which is much higher than 20\%; hence, an analysis of Table 6 leads to a position not in favor of the equivalent beam model. Nevertheless, before rejecting it, one may note that the excitation contains frequency components outside the frequency band chosen to be used for the estimation process which has been just performed and, up to this point, we have not yet validated the beam model in the frequency band of $[17.5,30] \mathrm{Hz}$. Hence, one might naturally ask: once the beam model has been given a certain level of credibility by the previous metrics, how accurate would it be within the frequency band [17.5, 30] Hz? More specifically, what's the capability of the proposed beam model to predict the dynamic behavior of the conductor Grosbeak in the frequency band [17.5, 30] Hz? In order to answer this question, we thus compare the measured and estimated FRFs for the three accelerometers in the frequency band of $[17.5,30] \mathrm{Hz}$ using the previous estimates for $E I$ and $\alpha$ shown in Table 4 . Figure 8 shows the magnitude of the APCC validation metric for the accelerometer AC1 in the frequency band of $[17.5,30] \mathrm{Hz}$.
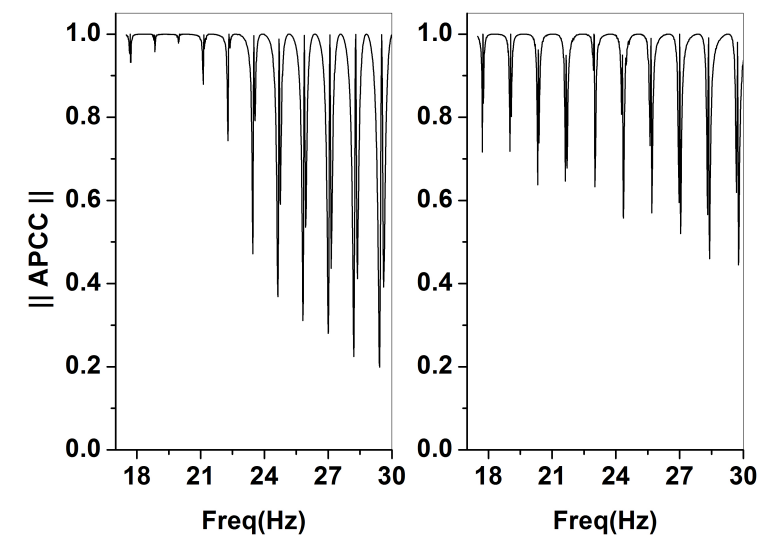

Figure 8. Magnitude of the APCC validation metric for the accelerometer $A C 1$ in the frequency band of $[17.5,30] \mathrm{Hz}$ using the parameter estimates computed at the first stage of the estimation process: (left) $T=16481 \mathrm{~N}$; (right) $T=21778 \mathrm{~N}$.

Comparing Figs. 4 and 8 one may note a significant deterioration of the predictability level of the beam model, mainly in the highest frequencies; the magnitudes of the APCC are drastically reduced for the majority of the frequency data points. Such deterioration in predictability explains the large values obtained for $C_{S G}$ in Table 6, what clearly indicates a demand for either a model revision or more data acquisition for the parameter estimation process. Therefore, in order to increase the predictability level of the model in the frequency band $[17.5,30] \mathrm{Hz}$ we proceed to the second stage of the estimation process.

\section{Second stage}

The second stage comprises the parameter estimation based on the measured FRFs of the accelerometers $\mathrm{AC} 2$ and $\mathrm{AC} 3$ in the frequency band of $[17.5,30] \mathrm{Hz}$. Two possible models are analyzed here and, henceforth, they are referred to as the 2-parameter and 3parameter models. For the 2-parameter model, the unknown parameters are the bending stiffness $E I$ and the aerodynamic damping coefficient $\alpha$, the material damping $\xi I$ is constant and equal to $10^{-4} \mathrm{Nsm}^{2}$ as previously done for the first stage. For the 3parameter model, the unknown parameters are the bending stiffness $E I$, the aerodynamic damping coefficient $\alpha$ and the material damping $\xi I$. The following parameterization is chosen: $E I=p_{1} \times 10^{3}, \alpha=p_{2}$ and $\xi I=p_{3} \times 10^{-2}$ (only for the 3-parameter model). The previous estimates for $E I$ and $\alpha$ shown in Table 4 provide the $a$ priori information for $p_{1}$ and $p_{2}$. Since no information is available for $\xi I$, we adopt a small value for the mean value of $p_{3}$ $\left(10^{-2}\right)$ and a large value for its standard deviation $\left(10^{5}\right)$. The estimated parameters are shown in Tables 7 and 8. 
Table 7. Estimates for the bending stiffness $E I=p_{1} \times 10^{3}$, the aerodynamic damping coefficient $\alpha=p_{2}$ and their corresponding standard deviations for the second stage $\left(\xi I=10^{-4} \mathrm{Nsm}^{2}\right)$.

\begin{tabular}{ccccc}
\hline$T[\mathrm{kgf}]$ & $p_{1}\left[\mathrm{Nm}^{2}\right]$ & $p_{2}\left[\mathrm{Nsm}^{-2}\right]$ & $\sigma_{p_{1}} / \sigma_{n}$ & $\sigma_{p_{2}} / \sigma_{n}$ \\
\hline 1680 & 0.5629 & 0.6228 & $4.1 \times 10^{-4}$ & 0.0070 \\
2220 & 0.6795 & 0.4450 & $3.7 \times 10^{-4}$ & 0.0055 \\
\hline
\end{tabular}

Table 8. Estimates for the bending stiffness $E I=p_{1} \times 10^{3}$, the aerodynamic damping coefficient $\alpha=p_{2}$ and the material damping $\xi I=p_{3} \times 10^{-2}$, and their corresponding standard deviations for the second stage.

\begin{tabular}{ccccccc}
\hline$T[\mathrm{kgf}]$ & $p_{1}\left[\mathrm{Nm}^{2}\right]$ & $p_{2}\left[\mathrm{Nsm}^{-2}\right]$ & $p_{3}\left[\mathrm{Nsm}^{2}\right]$ & $\sigma_{p_{1}} / \sigma_{n}$ & $\sigma_{p_{2}} / \sigma_{n}$ \\
\hline 1680 & 0.5344 & 0.3180 & 27.7026 & $9.6 \times 10^{-4}$ & $8.3 \times 10^{-5}$ & 0.8716 \\
2220 & 0.6788 & 0.3883 & 2.9638 & $4.4 \times 10^{-4}$ & 0.0098 \\
\hline
\end{tabular}

Figure 9 shows the magnitude of the APCC validation metric for the accelerometer AC1. For the tensile load $T=16481 \mathrm{~N}$, two important remarks should be highlighted. First, there has been an improvement in the predictability of the beam model, reflected in the APCC validation metric, when one compares Figs. 8 and 9. Second, the 2-parameter model seems to provide a better representation of the system within the frequency band of $[17.5,30] \mathrm{Hz}$ based on the APCC metric. For the tensile load $T=$ $21778 \mathrm{~N}$, there has also been an improvement in the predictability of the beam model when one compares Figs. 8 and 9; moreover, the 2-parameter and the 3-parameter models seem to be quite similar for the frequency range $[17.5,30] \mathrm{Hz}$. To close up the discussion, one might finally ask to what extent the second stage of the estimation process has affected the predictability of the beam model in the frequency band of $[5,17.5] \mathrm{Hz}$. To answer this question, another validation process has been performed by considering the time-domain accelerations measured during the tests performed with the conductor Grosbeak for both tensile loads.

For the tensile load $T=16481 \mathrm{~N}$, we consider the measured time-domain accelerations for a white noise excitation encompassing the frequency band of $[5,30] \mathrm{Hz}$. The Sprague and Geers' validation metrics computed for the 2-parameter and 3parameter models are indicated on Tables 9 and 10.

Table 9. Sprague and Geers validation metrics for comparison between measured and estimated time-domain responses under a white noise excitation encompassing the frequency band of [5,30] $\mathrm{Hz}$ (2-parameter model for $T=16481 \mathrm{~N}$ )

\begin{tabular}{ccccc}
\hline Accelerometer & $M_{S G}$ & $P_{S G}$ & $C_{S G}$ & $\left|\mathbf{q}^{\text {est }}\right|_{N}$ \\
\hline AC1 & -0.1267 & 0.1108 & 0.1683 & 0.3980 \\
AC2 & -0.1288 & 0.1329 & 0.1809 & 0.4641 \\
AC3 & -0.1696 & 0.2023 & 0.2640 & 0.7155 \\
\hline
\end{tabular}

Table 10. Sprague and Geers validation metrics for comparison between measured and estimated time-domain responses under a white noise excitation encompassing the frequency band of $[5,30] \mathrm{Hz}$ (3-parameter model for $T=16481 \mathrm{~N}$ ).

\begin{tabular}{ccccc}
\hline Accelerometer & $M_{S G}$ & $P_{S G}$ & $C_{S G}$ & $\left|\mathbf{q}^{\text {est }}\right|_{N}$ \\
\hline AC1 & -0.0794 & 0.1466 & 0.1677 & 0.4834 \\
AC2 & -0.0872 & 0.1719 & 0.1928 & 0.5665 \\
AC3 & -0.2167 & 0.2579 & 0.3368 & 0.9325 \\
\hline
\end{tabular}

Three important remarks may be drawn from the results shown in Tables 9 and 10. First, the Comprehensive Error Factors are less than $20 \%$ for the accelerometers $\mathrm{AC} 1$ and $\mathrm{AC} 2$ whereas the largest $C_{S G}$ values are computed for the accelerometer AC3. Second, the Comprehensive Error Factors are slightly lower for the 2-parameter model. Third, the point-to-point error norms $\left|\mathbf{q}^{\text {est }}\right|_{N}$ are significantly lower for the 2-parameter model. Figure 10 plots the experimental and estimated time histories for the accelerometer $\mathrm{AC} 1$ for the 2and 3-parameter models.
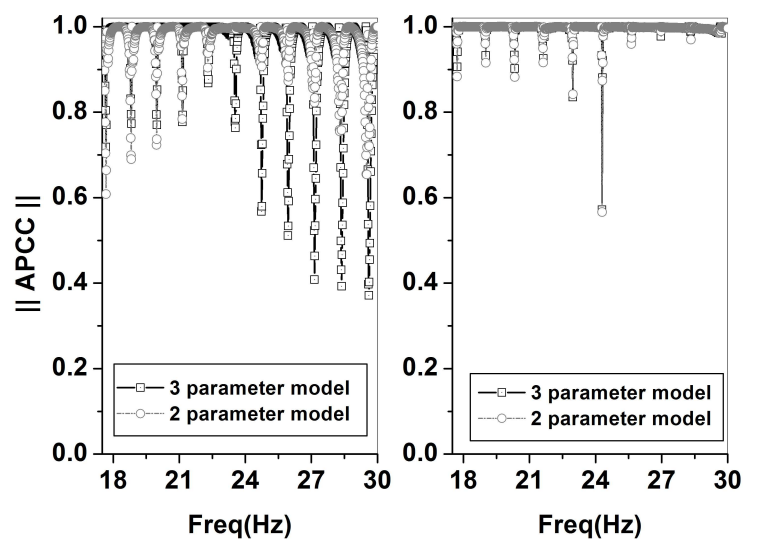

Figure 9. APCC validation metric for the accelerometer AC1 in the frequency band of $[17.5,30] \mathrm{Hz}$ : (left) $T=16481 \mathrm{~N}$ and (right) $T=21778 \mathrm{~N}$.

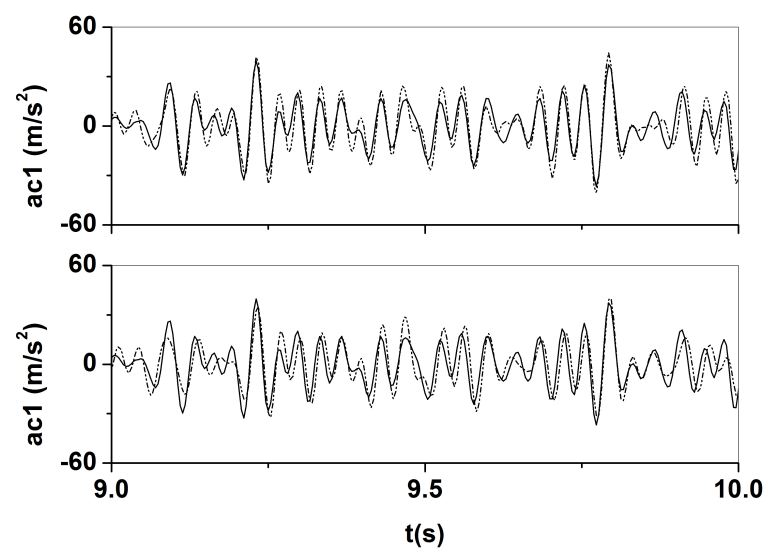

Figure 10. Time domain validation for the accelerometer AC1 for $T=16481 \mathrm{~N}$ and white noise excitation encompassing $[5,30] \mathrm{Hz}$ : (top) 2parameter model; (bottom) 3-parameter model. Black line: Experimental. Dotted Line: Estimated. 
For the tensile load $T=21778 \mathrm{~N}$, we consider the measured time-domain accelerations for a sine sweep excitation encompassing the frequency band of $[5,20] \mathrm{Hz}$. Tables 11 and 12 present the Sprague and Geers' validation metrics computed for the 2-parameter and 3-parameter models.

Table 11. Sprague and Geers validation metrics for comparison between measured and estimated time-domain responses under a sine sweep excitation encompassing the frequency band of $[5,20] \mathrm{Hz}$ (2-parameter model for $T=21778 \mathrm{~N}$ ).

\begin{tabular}{ccccc}
\hline Accelerometer & $M_{S G}$ & $P_{S G}$ & $C_{S G}$ & $\left|\mathbf{q}^{\text {est }}\right|_{N}$ \\
\hline AC1 & 0.0390 & 0.0877 & 0.0960 & 0.2722 \\
AC2 & 0.0579 & 0.1343 & 0.1462 & 0.4107 \\
AC3 & -0.1096 & 0.2807 & 0.3013 & 0.9128 \\
\hline
\end{tabular}

Table 12. Sprague and Geers validation metrics for comparison between measured and estimated time-domain responses under a sine sweep excitation encompassing the frequency band of $[5,20] \mathrm{Hz}$ (3-parameter model for $T=21778 \mathrm{~N}$ ).

\begin{tabular}{ccccc}
\hline Accelerometer & $M_{S G}$ & $P_{S G}$ & $C_{S G}$ & $\left|\mathbf{q}^{\text {est }}\right|_{N}$ \\
\hline AC1 & 0.0373 & 0.0814 & 0.0895 & 0.2530 \\
AC2 & 0.0839 & 0.1009 & 0.1313 & 0.3131 \\
AC3 & -0.0748 & 0.2585 & 0.2691 & 0.8253 \\
\hline
\end{tabular}

Based on the results indicated on Tables 11 and 12 one may draw the following conclusions: (i) the Comprehensive Error Factors are less than $15 \%$ for the accelerometers $\mathrm{AC} 1$ and $\mathrm{AC} 2$; the largest $C_{S G}$ values are obtained for the accelerometer $\mathrm{AC} 3$, placed at the driving point; (ii) the Comprehensive Error Factors are slightly lower for the 3-parameter model; (iii) the point-to-point error norms $\left|\mathbf{q}^{\text {est }}\right|_{N}$ are significantly lower for the 3-parameter model. Figure 11 plots the experimental and estimated time histories for AC2 for the 2- and 3-parameter models.
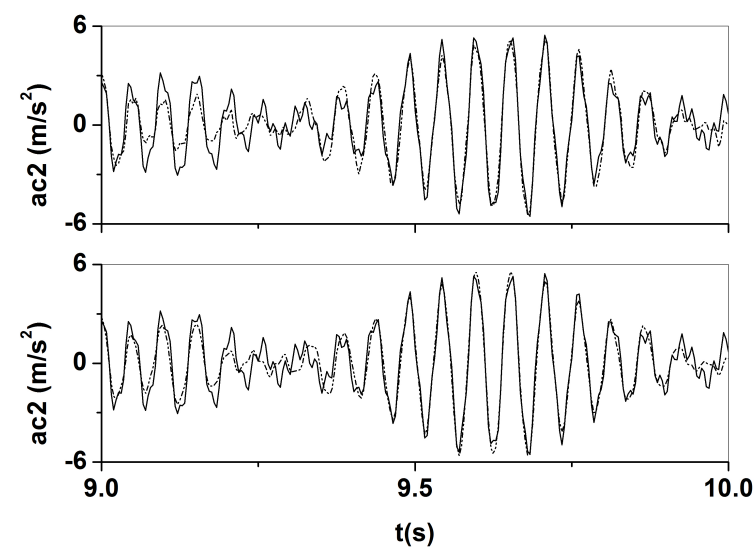

Figure 11. Time domain validation for the accelerometer AC2 for $T=21778 \mathrm{~N}$ and a sine sweep excitation encompassing [5, 20] Hz: (top) 2parameter model; (bottom) 3-parameter model. Black line: Experimental. Dotted line: Estimated.

Two important conclusions may be extracted from the results reported in the current work. First, the equivalent beam model with $E I$ and $\alpha$ given in Table 7 and with $\xi I=10^{-4} \mathrm{Nsm}^{2}$ or with $E I, \alpha$ and $\xi I$ given in Table 8 reproduces quite well the dynamic behavior of the conductor Grosbeak in the frequency range from $5 \mathrm{~Hz}$ to $30 \mathrm{~Hz}$, in both frequency and time domains for the tensile load $T=21778 \mathrm{~N}$. Second, the 2-parameter equivalent beam model with $E I$ and $\alpha$ given in Table 7 and with $\xi I=10^{-4} \mathrm{Nsm}^{2}$ seems to reproduce the dynamic behavior of the conductor Grosbeak in the frequency range from $5 \mathrm{~Hz}$ to $30 \mathrm{~Hz}$ better than the 3-parameter model for the tensile load $T=16481 \mathrm{~N}$.

\section{Final Remarks}

In the present work a computational model for a stranded cable typically used for transmission lines has been built. The computational model was built based on the basic principles of $\mathrm{V} \& \mathrm{~V}$ and the model tracking progress was guided by a pool of validation metrics suitable for experimental data commonly used in structural dynamics.

The experimental data were recorded during vibration tests performed with the conductor Grosbeak at CEPEL's laboratory span under two different tensile loads and with low sag-to-span ratios. The experimental data used for the parameter estimation process were the FRFs of the accelerometers AC2 and AC3. As transmission line conductors possess dense frequency spectra we decided to perform the estimation processes in two stages which are associated to two disjoint frequency bands. The parameter estimates based on the frequency band $[5,17.5] \mathrm{Hz}$ were taken into account for the parameter estimation based on the frequency band $[17.5,30] \mathrm{Hz}$ through the use of a maximum a posteriori objective function. We relied on model validation principles to assess the suitability of the equivalent beam model. The tracking progress of the model and its predictive capacity were quantitatively assessed by three validation metrics, namely: amplitude-phase correlation coefficient (APCC), Sprague and Geers metrics and a point-to-point error norm. It was considered time and frequency domain measured data for the analysis. The validation process provided favorable positions for the model for the set of available experimental data.

The results presented in this work are quite compelling due to the fact that the proposed approach is easy-handled. Furthermore, the model calibration based on validation metrics that has been presented in this work is broadly applicable to any structural system for which we can collect experimental dynamic data. As a final comment, we believe that the proposed equivalent homogeneous beam model used in this work is able to predict the dynamic behavior of the conductor Grosbeak measured on laboratory in both frequency and time domains. Hence, we expect that it may be useful for computer simulations of aeolian vibrations, at least for frequency ranges expected for those vibrations and for tensile loads commonly encountered in the field.

\section{Acknowledgements}

We would like to thank Mr. José Martins Ferreira, Mr. Belchior Reis Neto and Mr. Antônio Carlos de Andrade e Silva (B.Sc., Electrical Engineer) for all their help during the tests performed at CEPEL's laboratory span. We also would like to thank Miss Bianca Walsh from IBGE for her careful reading of the manuscript and for her improved comments and suggestions. The authors would like to thank the financial support provided by the National Council of Scientific and Technological Development $(\mathrm{CNPq})$ and by Fundação Carlos Chagas Filho de Amparo à Pesquisa do Estado do Rio de Janeiro (FAPERJ) due to the project E-26/111.599/2010-APQ1.

\section{References}

Adhikari, S., 2000, "Damping Models for Structural Vibrations", Ph.D. Thesis, Cambridge University, Cambridge, UK, 204 p.

American Institute of Aeronautics and Astronautics, 1998, "Guide for the Verification and Validation of Computational Fluid Dynamics Simulations", Technical Report No. AIAA-G-077-1998. 
American Society of Mechanical Engineers, 2006, "Guide for the Verification and Validation in Computational Solid Mechanics", Technical Report No. ASME V\&V 10-2006.

Barbieri, N., Junior, O.H.S. and Barbieri, R., 2004, "Dynamical analysis of transmission line cables. Part 1 - Linear Theory", Mechanical Systems and Signal Processing, Vol. 18, pp. 659-669.

Beck, J.V., 2003, "Sequential Methods in Parameter Estimation", Chapter 1 of Inverse Engineering Handbook, Edited by Keith A. Woodbury, CRC Press, 466 p.

Cardou, A., 2006, "Taut helical strand bending stiffness", http://www.utfscience.de.

Castello, D.A. and Matt, C.F., 2007, "Estimation of bending stiffness and damping of transmission line conductors", Proceedings of the Inverse Problem, Design and Optimization Symposium, Miami, Florida, USA, April 16-18, Paper IPDO-068.

CIGRÉ Scientific Committee 22, Working Group 01, 1989, "Report on Aeolian Vibrations", Electra, Vol. 124, pp. 41-77.

Claren, R. and Diana, G., 1969, "Mathematical Analysis of Transmission Line Vibration", IEEE Transactions on Power Apparatus and Systems, Vol. 88, No. 12, pp. 1741-1771.

Crandall, S.H., 1970, "The Role of Damping in Vibration Theory", Journal of Sound and Vibration, Vol. 11, No. 1, pp. 3-18.

Dhotarad, M.S., Ganesan, N. and Rao, B.V.A., 1978, "Transmission Line Vibration with 4R Dampers", Journal of Sound and Vibration, Vol. 60, No. 4, pp. 604-606.

Diana, G., Falco, M., Cigada, A. and Manenti, A., 2000, "On the Measurement of Overhead Transmission Lines Conductor Self-Damping", IEEE Transactions on Power Delivery, Vol. 15, No. 1, pp. 285-292.

Sornette, D., Davis, A.B., Kamm, J.R. and Ide, K., 2008, "A general strategy for physics-based model validation illustrated with earthquake phenomenology, atmospheric radiative transfer and computational fluid dynamics", Computational Methods in Transport: Verification and Validation, Springer, Vol. 62, pp. 19-73, Ed. Frank Graziani.

Friswell, M.I., Garvey, S.D. and Penny, J.E.T., 1995, "Model Reduction Using Dynamic and Iterated IRS Techniques", Journal of Sound and Vibration, Vol. 186, No. 2, pp. 311-323.

Geers, T.L., 1984, "An objective error measure for the comparison of calculated and measured transient response histories", Shock Vibration Bulletin, Vol. 54, pp. 99-107.

Greenwald, M., 2010, "Verification and Validation of Magnetic Fusion”, Physics of Plasma, Vol. 17, No. 058101, pp. 1-7.

Hagedorn, P., 1982, "On the computation of damped wind-excited vibrations of overhead transmission lines", Journal of Sound and Vibration, Vol. 83, No. 2, pp. 253-271.

Hagedorn, P., Schmidt, J. and Nascimento, N., 1987, "Stochastic Field Processes in the Mathematical Modelling of Damped Transmission Line Vibrations", Proceedings of the 5th Conference on Mathematical Modelling, Berkeley, USA.
Hemez, F., Atanturktur, H.S. and Unal, C., 2010, "Defining Predictive Maturity for Validated Numerical Simulations", Computers and Structures, Vol. 88, pp. 497-505.

Hughes, T.J.R., 2000, "The Finite Element Method: Linear Static and Dynamic Finite Element Analysis", Dover Publications, New York, 672 p.

Irvine, H.M., 1981, "Cable Structures", The MIT Press Series in Structural Mechanics, Vol. 1, MIT Press, Cambridge, MA, 259 p.

Kim, B.H. and Park, T., 2007, "Estimation of Cable Tension Force Using the Frequency-Based System Identification", Journal of Sound and Vibration, Vol. 304, pp. 660-676.

Liang, Y., Tam, K.-S. and Broadwater, R., 2010, "Load Calibration and Model Validation Methodologies for Power Distribution Systems", IEEE Transactions on Power Systems, Vol. 25, No. 3, pp. 1393-1401.

Matt, C.F. and Castello, D.A., 2007, "On the numerical identification of the bending stiffness and the damping of transmission line conductors", Proceedings of the XII International Symposium on Dynamic Problems of Mechanics, Ilha Bela, SP, Brazil, February 26 - March 02.

Meynen, S., Verma, H., Hagedorn, P. and Schäfer, M., 2005, "On the numerical simulation of vortex-induced vibrations of oscillating conductors", Journal of Fluids and Structures, Vol. 21, pp. 41-48

Oberkampf, W.L. and Barone, M.F., 2006, "Measurements of agreement between computation and experiment: validation metrics", Journal of Computational Physics, Vol. 217, pp. 5-36.

Orlande, H.B.R., 2002, "Parameter and Function Estimation Problems in Heat and Mass Transfer", Proceedings of Fifth World Congress on Computational Mechanics, Vienna, Austria, July 7-12, Eds. H.A. Mang, F.G. Rammerstorfer and J. Eberhardsteiner.

Özișik, M.N. and Orlande, H.R.B., 2000, "Inverse Heat Transfer: Fundamentals and Applications", Taylor and Francis.

Rawlins, C.B., 1979, "Transmission Line Reference Book: WindInduced Conductor Motion”, Electric Power Research Institute (EPRI), Palo Alto, California

Rawlins, C.B., 1983, "Model of Power Imparted to a Vibrating Conductor by Turbulent Wind", Technical Report, ALCOA conductor products company technical note No. 31 .

Schwer, L.E., 2007, "Validation Metrics for Response Histories: Perspectives and Case Studies", Engineering with Computers, Vol. 23 , pp. 295-309.

Sih, G.C., Tang, X.S., Li, Z.X., Li, A.Q. and Tang, K.K., 2008, "Fatigue Crack Growth Behavior of Cables and Steel Wires for the Cable-Stayed Portion of Runyang Bridge: Disproportionate loosening and/or tightening of Cables", Theoretical and Applied Farcture Mechanics, Vol. 49, pp. 1-25.

Sprague, M.A. and Geers, T.L., 2003, "Spectral elements and field separation for an acoustic fluid subject to cavitation", Journal of Computational Physics, Vol. 184, pp. 149-162.

Wang, X.Q., So, R.M.C. and Liu, Y., 2001, "Flow-Induced Vibration of an Euler-Bernoulli Beam", Journal of Sound and Vibration, Vol. 243, No. 2 , pp. 241-268. 